Vietnam Academy of Science and Technology
Vietnam Journal of Earth Sciences
(VAST)
Website: http://www.vjs.ac.vn/index.php/jse

\title{
Automatic detection of surface water bodies from Sentinel-1 SAR images using Valley-Emphasis method
}

\author{
Nguyen Ba Duy \\ University of Mining and Geology
}

Accepted 25 December 2015

\begin{abstract}
Surface water resource plays as an important role in human daily life and in the eco-environment. In the study Valley-Emphasis method of automatic water extraction was employed to identify surface water bodies at three study areas, having different landscapes and covers, using Sentinel-1A IW images widely used automated Otsu method was performed for extracting surface water bodies to compare proposed method. The results of proposal method were compared to those of widely used Otsu method and the reference data (e.g. Lansat 7, 8) gave the highest Completeness (User accuracy), Correctness (Producer accuracy) and Quality (Overall accuracies) at 98.8\%, $90.7 \%$ and $89.7 \%$, respectively. The employed method is straightforward, easy to implement and may be applied for other areas even at regional or global scales. The method also improves automatic identification level of surface water bodies, providing essential information for flood disaster research.
\end{abstract}

Keyword: Surface Water body, Valley-Emphasis Algorithm, Sentinel-1, SAR.

C2015 Vietnam Academy of Science and Technology

\section{Introduction}

The ability to map open surface freshwater (i.e. lakes, ponds, reservoirs) is integral to many hydrologic and agricultural models, wildlife management programs, and recreational and natural resource studies (Henderson, 1995). Concomitantly, the visibility and appearance of open surface water bodies should have supplemental value in radar geoscience research addressing the relationships among radar wavelength, specular reflectance, smooth/rough signal return criteria, and terrain features, especially when spatial variables are considered. Open surface water is a landscape feature that is generally regarded as easily detected on radar imagery; water surfaces are considered to be specular reflectors of radar energy, scattering the

Email: nguyenbaduy@humg.edu.vn incident radar signal away from the antenna. As a consequence, open surface water generally appears in dark tone or black colour, no return signal on the imagery. However, this observation is an oversimplification. The radar signal is sensitive to surface roughness and water will not always appear as, or actually be; a smooth surface. Other surfaces can also appear smooth at radar wavelength and thus similar in appearance to surface water bodies. Which surfaces appear smooth and under what conditions depends on the surface and the wavelength of the radar systems, shadow effect for example. The appearance (radar signal response) of surface water bodies may or may not be similar in appearance to its background as a function of the system wavelength.

Due to the cloud penetration capability, SAR satellites are almost independent from weather and day/night acquisition capabilities. Therefore, they 
N.B. Duy/Vietnam Journal of Earth Sciences 37 (2015)

are more suitable than optical sensors to reliably and timely map inundated areas in flood situations, which usually occur under overcast sky conditions, especially in tropical climate zones, as Vietnam for example. SAR sensors have been paid more attention in the open water bodies mapping, flood monitoring, as well as disaster assessment. Since nearly two decades, operational space borne systems like ERS-1/2, Envisat ASAR and Radarsat-1 have been used to map water bodies and flood situations at C-Band wavelength independent of cloud coverage with medium resolution. The ALOS PALSAR sensor provides the possibility to study water features at L-Band wavelength since its launch in 2006. Recent development in microwave remote sensing shows the advantages of several high-resolution SAR sensors. At June 15, 2007 the German X-Band TerraSAR-X satellite was launched. At December 14, 2007 the Canadian C-Band Radarsat-2 satellite was also launched. Sentinel-1A was launched on 3 April 2014 on a Soyuz rocket from Europe's Spaceport in French Guiana. Comparing with the world's leading space-borne radar system Sentinel1A has a new breakthrough on the spatial resolution, polarization, incident angle and width. Sentinel-1A can gain horizontal, vertical and a variety of polarization combination information, which can provide products with different angles and a variety of spatial resolution products and provide greater coverage and shorter repeated observation because of its scanning and global monitoring; these features make it becomes the most powerful Space-borne Radar system. Main orbit characteristics of Sentinel-1A are sunsynchronous, near-polar, and circular at a flying altitude of $693 \mathrm{~km}$. The satellite features a nominal repetition rate of 175 orbits in 12 days. The Sentinel-1 mission requirements indicate that one main operational imaging mode (Interferometric Wide-swath mode) in combination with the Wave mode satisfies most currently known service requirements, avoids conflicts and preserves revisit performance, provides robust and reliable service, simplifies mission planning, reduces operational costs and also satisfies future requests by building up a consistent long-term archive. Two mutually exclusive dual-polarization modes are provided for the imaging modes. Based on the mission requirements, the following main operational measurement modes are implemented: Interferometric Wide-swath mode (IW), Wave mode (WV); and for continuity reasons and emerging user requirements: Strip Map mode (SM), Extra Wide-swath mode (EW). The size of the ground track is either $20 \mathrm{~km}$ (WV) or $80 \mathrm{~km}$ $(\mathrm{SM})$ in azimuth and $20 \mathrm{~km}$ in range. In the Interferometric Wide-swath mode (IW), a scene has a swath width of $250 \mathrm{~km}$ in the Extra Wideswath mode (EW), a scene has a swath width of $410 \mathrm{~km}$. Depending on the incidence angle, and the ground resolution can be up to 5 meters. For each imaging mode, a variety of different acquisition parameters can be defined (e.g. incidence angle, polarization, processing parameters). The high resolution and the increased observation frequency of the new class of SAR sensors offer enormous potential in the domain of water bodies mapping as well as flood monitoring. However, the improved spatial resolution of the SAR data results in a large variety of very small-scaled image objects, which makes image processing and analysis even more challenging. The general aim of the ongoing research activities is applying the improved the Li's Minimum Cross Entropy Threshold Method to three study sites in northern Vietnam: the reservoir of Thac Ba hydroelectric, Red River, and Thai Binh River.Water body information extraction achieved relatively good results.

Image thresholding is a popular technique for image segmentation because of its simplicity and computational efficiency. The basic idea of image thresholding is to automatically select an optimal gray-level threshold value based on gray-level distribution, and compare each pixel in the image to the threshold value to separate objects of interest in an image from the background. Because of its wide applicability to other areas of image processing and applications, many automatic thresholding algorithms have been proposed in the literatures. Among the image thresholding techniques, the Otsu method is one of the better threshold selection methods for general real world images with respect to uniformity and shape measures (Otsu N, 1979). This method selects threshold values that maximize the between-class variances of the histogram. The Otsu method assumes that the gray level of the object and the background in an image distribution is Gaussian distribution with equal variances, thus it is optimal 
Vietnam Journal of Earth Sciences 37 (2015) 328-343

for thresholding a histogram with bimodal or multimodal distribution but fails if the histogram is unimodal or close to unimodal. Fuang revised the Otsu method for selecting optimal threshold values for both unimodal and bimodal distributions (Fuang $\mathrm{Ng}$, 2006). The method, called valley-emphasis method, uses similar objective function as the Otsu method but give more weights to the valley points in the histogram. In other words, the valley-emphasis method favors values that reside at the valley of two peaks, or at the bottom rim of a single peak. Therefore, the valley-emphasis method is able to select optimal threshold values for both bimodal and unimodal distributions. Recently, Lun and Bo pointed out that the weighting effect of the valleyemphasis method might not be strong enough for cases where the variance of the object is very different from that of the background and thus fails to locate correct threshold value (Lun F and Bo L, 2012). They suggested that including the neighboring values around the valley points could improve the weighting effect. However, it is unclear how to determine the appropriate size for the neighborhood to achieve optimal thresholding results.

In this study, I proposed an approach for improving the valley-emphasis method for image thresholding by introducing a Gaussian weighting scheme in the objective function to enhance the weighting effect.

\section{Study area and Data acquisition}

\subsection{Study area}

Three study sites were selected to perform the research which covered entire or a part of Thac $\mathrm{Ba}$ Lake, Yen Bai province (Study side 1), Nui Coc Lake (Thai Nguyen province), Red River (Ha Noi, Vinh Phuc), Dong Mo Lake (Ha Noi) (Study site 2), and Thai Binh River (Thai Binh Hai Phong), shrimp farming, coastal zone and a part of East sea (Study side 3).

The first study site, Thac Ba Lake is one of three Vietnam's largest artificial lakes and famous for the first hydro power plant. Thac Ba Lake is also well known for its eco-system site. Thac $\mathrm{Ba}$ Lake was built in 1970 by the construction of a dam on the Chay River. Thac Ba reservoir covers a total area of 23400 hectares, of which 1905 hectares is water area and the remaining 435 hectares of islets on the Reservoir (Figure 1). This artificial lake has 1,331 islands and hills with various ecological environments. The water in the lake is imprinted with the reflections of the surrounding ancient forest creating a blue and clear background of the water.
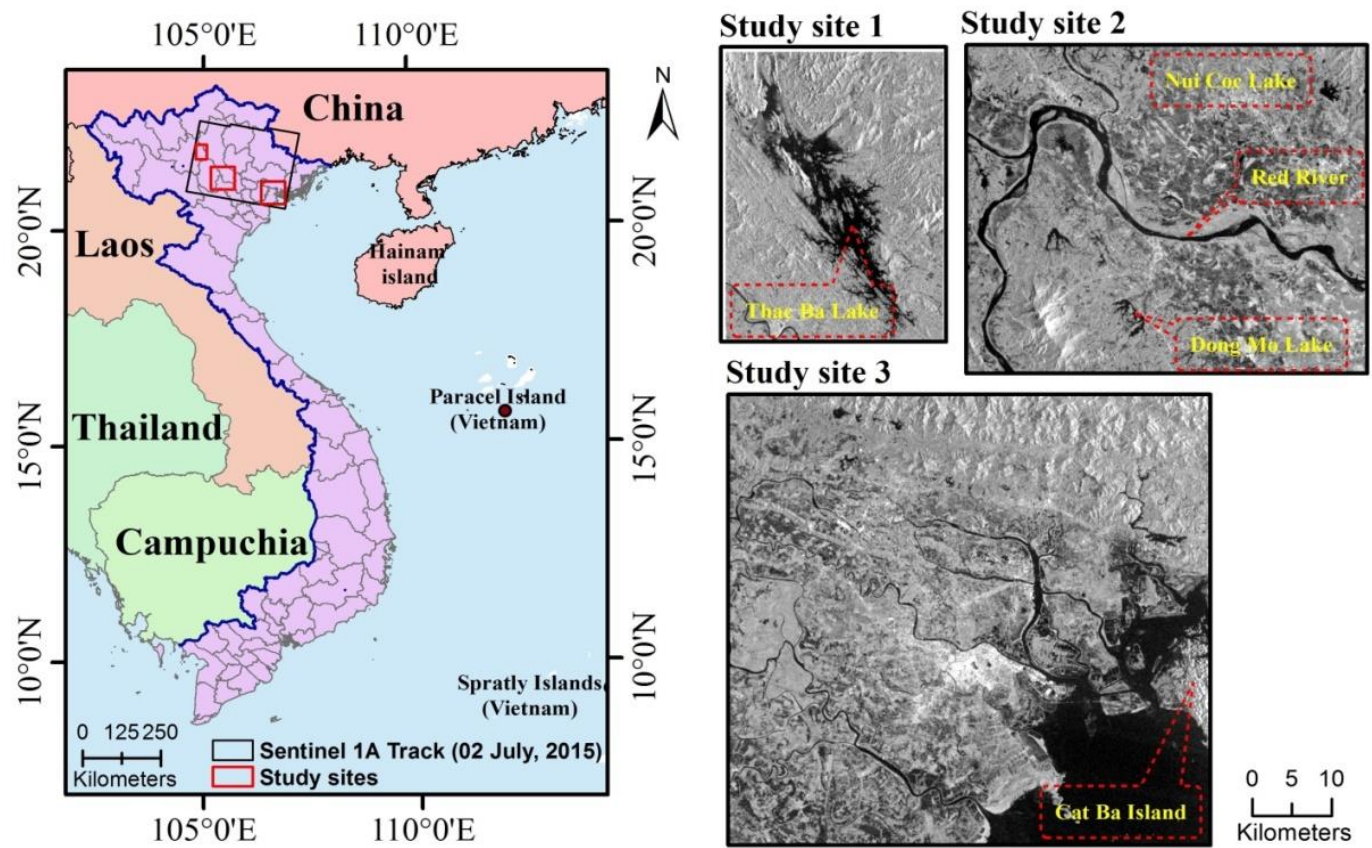

Fig. 1. Study sites 
N.B. Duy/Vietnam Journal of Earth Sciences 37 (2015)

The second study site is almost flat and hilly area with elevations ranging from 0 to $300 \mathrm{~m}$ above mean of sea level.

The third study site covered part of Hai Duong province and two eastern coastal provinces (Thai Binh and Hai Phong) in the Red River Delta region. Being a delta province with flat terrain and slope of below 1 percent; the terrain of Thai Binh and Hai Phong province runs downward from the North to the South and varies its height of 1 to $2 \mathrm{~m}$ to the sea level. An even and flat terrain, there is no hills and mountains, several faces border river and one face border sea.

\subsection{Remote sensing data}

\subsubsection{Microwave remote sensing data (Sentinel 1 SAR data)}

The study has used the satellite imagery of Sentinel 1A (dual polarization VV and VH) with $10 \mathrm{~m}$ spatial resolution in July 02,2015 . The satellite data used in the present study are showed in the Figure 2.
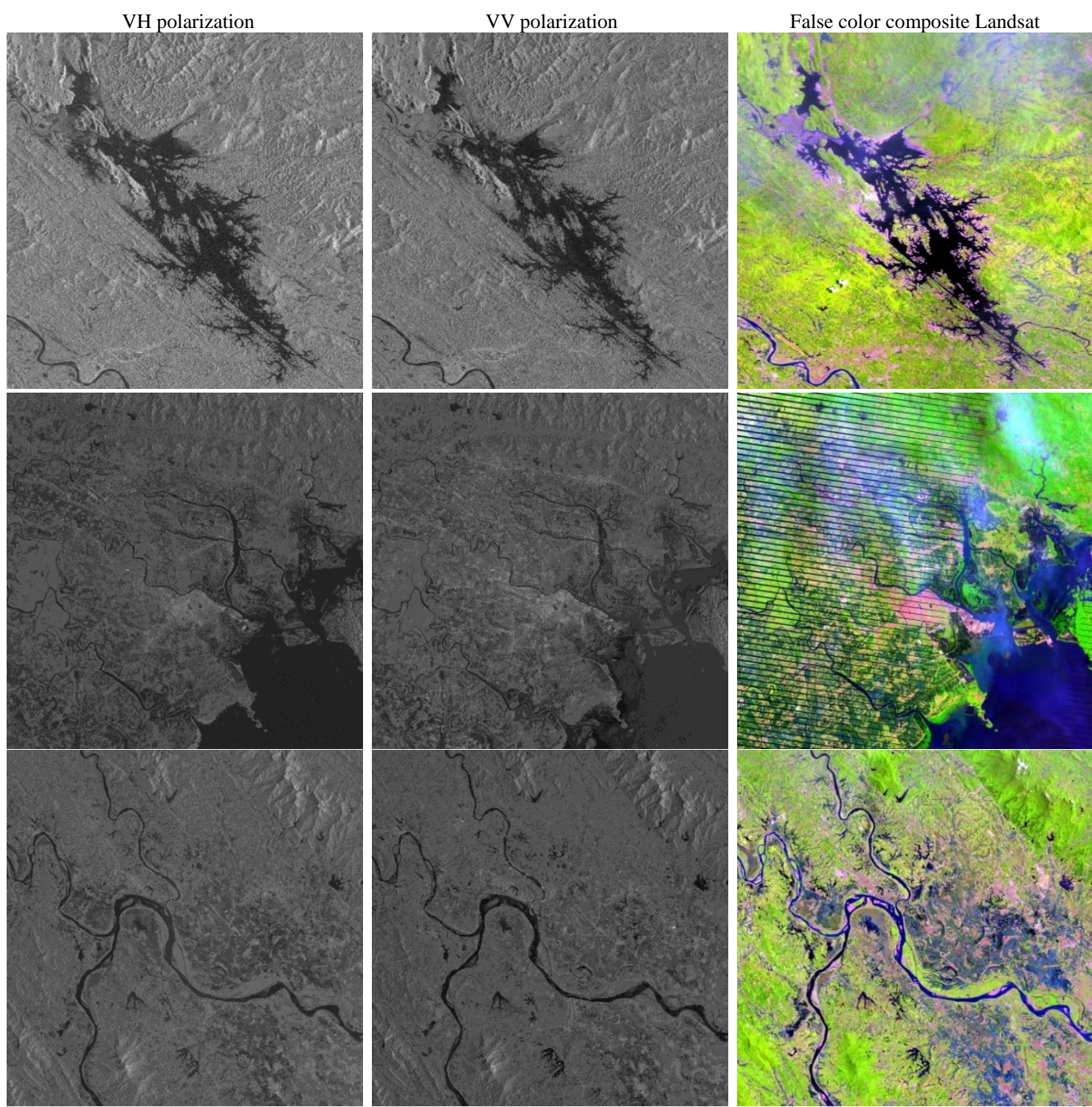

Fig. 2. The satellite data used in the present study: Sentinel $1 \mathrm{~A}$ SAR data acquired in July 02, 2015 (a) VV image, (b) VH image; False color composite Landsat 8 acquired July 01, 2015; and False color composite Landsat 7 acquired July 2, 2015 
2.2.2. Optical remote sensing data (LANDSAT 7 and LANDSAT 8 data)

In this study, optical remote sensing data (LANDSAT 7 and LANDSAT 8) were collected from http://earthexplorer.usgs.gov/ website. Due to very dynamic change of the surface water bodies and free cloud available data reason, the acquisition of these two datasets were collected in the same day and one day after Sentinel SAR data acquisition. There is one LANDSAT 7 scene acquired in the same date with Sentinel 1A SAR scene (02 July, 2015) and cover entire study site number 1 and study site number 2 . The other LANDSAT 8 scene acquired one day before Sentinel 1A SAR scene (01 July, 2015) and cover entire study site number 3. The atmospheric correction was carried out using the MOTRAN 4 model, which is embedded in the ENVI/FLAASH module applied atmospheric correction for these two optical dataset, in order to derive the ground surface reflectance (GSR). Then, surface water bodies were classified by using support vector machine algorithm (ENVI module). These results are used later for validation the results (surface water bodies) that derive from Sentinel 1A SAR.

\section{Methodology}

\subsection{Satellite image processing}

According to the Rayleigh criterion, the incidence angle decreases that the roughness of target surface increases, which will make the range of target scattering energy distribution expanded. When the surface of the water generated waves due to strong winds, that is equivalent to the increase in surface roughness, then the radar echo energy increases, and the rate weakened with the increase of the incident angle. When the incident angle decreased, the scattering coefficient of wavy surface increased, and it is very difficult to distinguish with build-up areas, therefore, we should do local incident angle normalization for the water body identification. In this paper, we applied flattening gamma for radiometric terrain correction for Sentinel-1A SAR data (Small, 2011). When the surface is rough, echo has nothing to do with the incident angle and polarization. But when the surface is smooth, echo is related to the polarization in a certain extent. For the same surface, the measured value of crosspolarization $(\mathrm{VH})$ is smaller than co-polarization (VV). In this study we observed that $\mathrm{VH}$ image still provides useful information for water extraction. Therefore, for the Sentinel-1A data used to disaster evaluation, we can use both $\mathrm{VH}$ and VV images products. Based on the above discussion, in this paper, the input data is Sentinel1A data in the July 02, 2015 for three study sites (Figure 1).

\subsubsection{Calculating Backscattering Coefficient (Gamma nought $-\gamma^{o}$ )}

For water body mapping as well as flood disaster assessment, we must accurately identify the water body pixel. And for the water body extraction from Sentinel-1A images mainly based on that water has lower backscattering characteristics in the microwave range. Using the Sentinel-1 Toolbox software provided by ESA to calculate backscattering coefficient, the main contents include original data extraction, clip image, backscattering image generation. Processing Sentinel-1A data according the following steps: import data, subset data, data calibration, backscattering image generation, then the backscattering coefficient (gamma nought $\gamma^{o}$ ) of each pixel will be obtained, so are data speckle filtering and data terrain correction (Small, 2011).

\subsubsection{Removing the Noise}

Since SAR is a coherent-imaging system. So the speckle noise inevitably exists in the images. Removal of the speckle noise is very important to obtain high-quality radar images. Previous studies confirmed that SAR images comply with the multiplicative noise model from theoretical deduction and test., and used the multiplicative noise model filtering algorithm with different filters and different window sizes to filter the ASAR images. The results showed that with $7 \times 7$ window Enhanced Lee had the best effect (Figure 3). Therefore, this paper uses $7 \times 7$ window Enhanced Lee filtering method provided with Sentinel-1 Toolbox version 1.1 for the Sentinel-1A image filter processing (Gonzalez $\mathrm{C}$ and Woods $\mathrm{R}$, 2002), (Ye Z et al., 2008). 
N.B. Duy/Vietnam Journal of Earth Sciences 37 (2015)

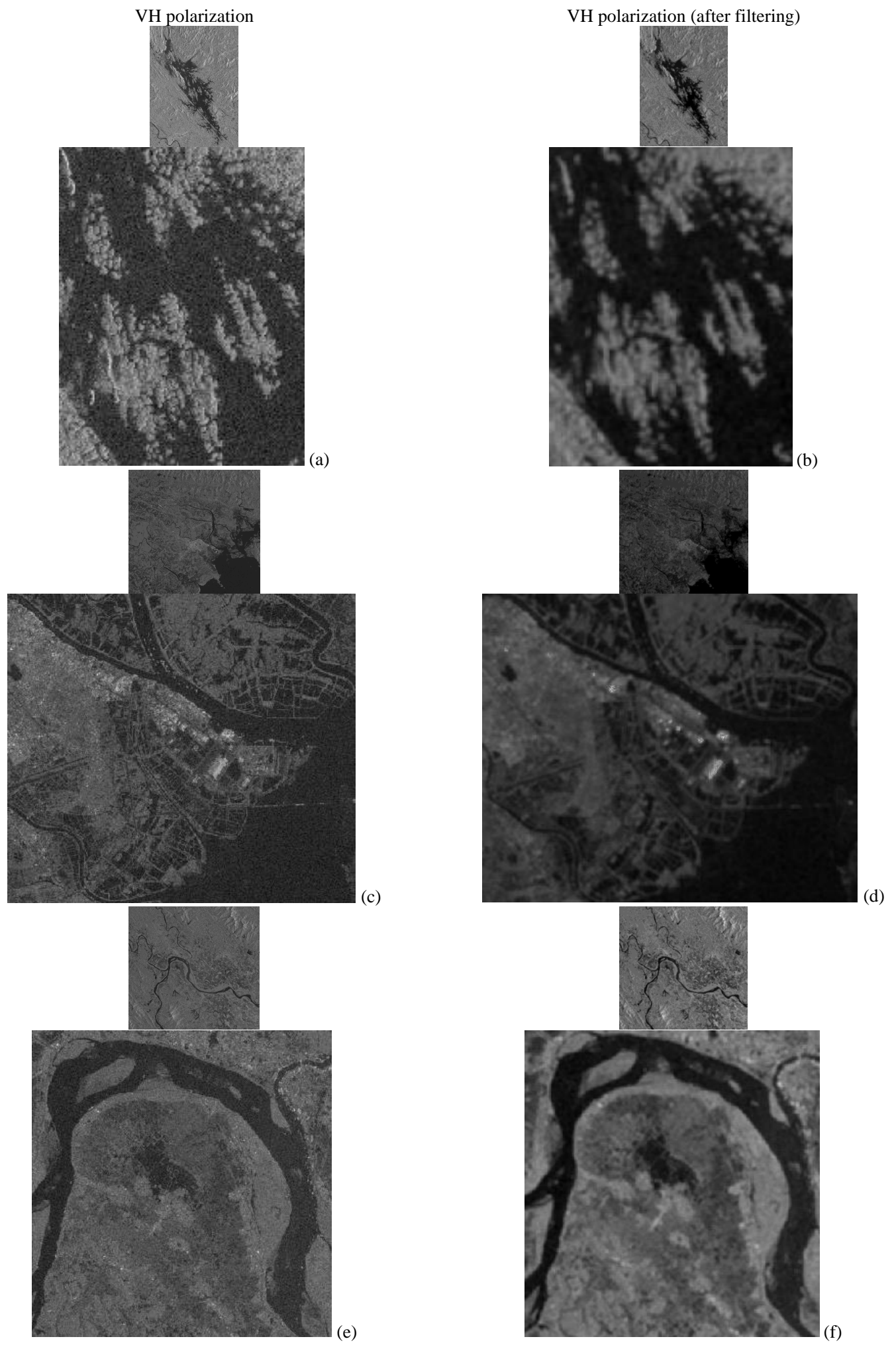

Fig. 3. The backscattering coefficient map before and after filtering 
Vietnam Journal of Earth Sciences 37 (2015) 328-343

\subsection{Image Threshold methods for water body extraction}

For the flood disaster assessment, the main issue is the water area information extraction; the process is actually "Binarization" process in the image segmentation. Therefore, the threshold method is often used in water body extraction. Currently, image thresholding is considered one of the most popular methods to segment an image and is commonly used for separating objects from the background (Zhang et al., 2010). Although it seems simple the problem of choosing a good and an accurate threshold value is a difficult task. The most widely used thresholding technique based on the grey level histogram. When an image, $\mathrm{f}(\mathrm{x}, \mathrm{y})$, is composed of dark objects on a light background, then the foreground is clearly distinguishable from the background and this case the image histogram will be bimodal so that the threshold value will lie in the valley of the histogram ensuring that the objects can be extracted. Any pixel $(x, y)$ for which $\mathrm{f}(\mathrm{x}, \mathrm{y})>\mathrm{T}$ (threshold) is considered as belonging to the object class, otherwise, it belongs to the background class (Gonzalez C and Woods R, 2002). Unfortunately, this is not the case in most images. This has led some researchers to develop techniques to transform the image histogram into a bimodal form. In addition to histogram based thresholding techniques, several techniques have been proposed, trying to find a way of choosing the best value of threshold (T) that will result in an accurate segmentation. Hui-Fuang revised the Otsu method for selecting optimal threshold values for both unimodal and bimodal distributions (Fuang $\mathrm{Ng}$, 2006). The method, called valley-emphasis method, uses similar objective function as the Otsu method but give more weights to the valley points in the histogram. In other words, the valleyemphasis method favors values that reside at the valley of two peaks, or at the bottom rim of a single peak. Therefore, the valley-emphasis method is able to select optimal threshold values for both bimodal and unimodal distributions. However, it is unclear how to determine the appropriate size for the neighborhood to achieve optimal thresholding results. In the following subsection, a brief introduction review the Otsu method is presented (3.2.1); while sub-section (3.2.2) and (3.2.3) present the valley-emphasis method and a modification for the valley-emphasis method for selecting optimal image threshold respectively.

\subsubsection{Principle of the Otsu method}

An image is represented by a 2 dimensions gray-level intensity function $f(x, y)$. The value belonging to location $(\mathrm{x}, \mathrm{y})$ represents by the function $\mathrm{f}(\mathrm{x}, \mathrm{y})$ is the gray-level, ranging from 0 to $\mathrm{L}-1$, where $\mathrm{L}$ is the number of distinct gray-levels. Let the number of pixels with gray-level $i$ be $n_{i}$, and $\mathrm{N}$ be the total number of pixels in a given image, the probability of occurrence of gray-level $\mathrm{i}$ is defined as $p_{i}=\frac{n_{i}}{N}$. Then the average gray-level of the entire image is computed as: $\mu_{T}=\sum_{i=0}^{L-1} i p_{i}$. In the single thresholding case, the pixels of an image are divided into two classes $\mathrm{C}_{1}=\{0,1, \ldots, \mathrm{t}\}$ and $\mathrm{C}_{2}=\{\mathrm{t}+1, \mathrm{t}+2, \ldots, \mathrm{L}-1\}$, where $\mathrm{t}$ is the threshold value. $\mathrm{C}_{1}$ and $\mathrm{C}_{2}$ are normally corresponding to the foreground (objects of interest, water bodies for example) and the background (none-water bodies for example). The probabilities of the two classes are: $\omega_{1}(t)=$ $\sum_{i=0}^{t} p_{i}$ and $\omega_{2}(t)=\sum_{i=t+1}^{L-1} p_{i}$. Based on these two keys value, the mean gray-level values of the two classes can be computed and then by using discriminant analysis, Otsu (Otsu N, 1979) showed that the optimal threshold $t^{*}$ can be determined by maximizing the between-class variance by below simplified equation:

$t^{*}=\operatorname{AgrMax}_{0 \leq t \leq L}\left\{\omega_{1}(t) \mu_{1}^{2}(t)+\omega_{2}(t) \mu_{2}^{2}(t)\right\}$

From (1) we can see that Otsu method is simple and easy to realize thus makes it one of the most commonly used threshold methods in engineer practices with satisfactory results. However, Otsu method is optimal for thresholding a histogram with bimodal or multimodal distribution but fails if the histogram is unimodal or close to unimodal.

\subsubsection{Principle of the Valley-Emphasis method}

In the case of single object, the idea threshold value should lie at the valley of the two peaks (bimodal), or at the bottom rim of a single peak (unimodal), as shown in Fig. 4f. Based on this observation, (Fuang Ng, 2006) proposed the valley emphasis method to select a threshold value with a small probability of occurrence $\left(p_{t}\right)$ which also maximizes the between-class variance. They 
N.B. Duy/Vietnam Journal of Earth Sciences 37 (2015)

introduced a weighting term which is defined as inverse proportional to $\mathrm{p}_{\mathrm{t}}$ : $\mathrm{W}(\mathrm{t})=1-\mathrm{p}_{\mathrm{t}}$. The optimal threshold is chosen by maximizing the revised objective function as:

$$
\begin{aligned}
& t^{*}=\operatorname{AgrMax}_{0 \leq t \leq L}\left\{W ( t ) \left(\omega_{1}(t) \mu_{1}^{2}(t)+\right.\right. \\
& \left.\left.\omega_{2}(t) \mu_{2}^{2}(t)\right)\right\}
\end{aligned}
$$

The first term in (10) is the weight and the second term is the between-class variance of the image. The smaller the $p_{t}$ value, the larger the weight will be. This weight ensures that the resulting threshold will be a value that resides at the valley or bottom rim of the gray-level distribution.

\subsection{Principle of the modified Valley-Emphasis method}

It was shown in (2) that the weighting term $\mathrm{W}(\mathrm{t})=1-\mathrm{p}_{\mathrm{t}}$ might not be significant enough for cases where the variance of the object is very different from that of the background and thus fails to determine correct threshold value. They suggested that including the threshold value could have derived from the weighting function. In this study, I propose a local maximum of weighting term scheme that efficiently uses the simple mathematical formulas for inclusion in the position of local minimum value from objective function. The optimal threshold is chosen by local maximizing of the objective function as: the revised objective function as:

$$
t^{*}=\operatorname{AgrMax}_{0 \leq t \leq L}\{W(t)\}
$$

\section{Results \& Discussion}

\subsection{Results}

We can obtain the backscattering coefficient image after pretreatment on Sentinel -1A SAR data. From these backscattering images, the optimal threshold was computed by using above three threshold algorithms. Less than the threshold, the pixel is the water, and more than the threshold, the pixel is not the water, as can be seen in the Figure 4 and Figure 5.

Figure 4 shows the histogram of entire three study sites image and the Otsu objective function before and after applying the valley weight. The threshold value selected by Otsu method is 92, which is not at the valley (about 63) of the histogram (study site 1 , figure $4 a$ ). The threshold value selected by the valley-emphasis method is 77 , which still deviate from the valley point. On the other hand, the modification Valley-Emphasis method reports a threshold value of 63 , which is close to the valley point of the histogram. A similarly properties can be observed from figure $4 \mathrm{~b}$ and figure $4 \mathrm{c}$ for the study site 2 and study site 3 respectively.

From the images, surface water bodies extraction results are worthy. However, there are a number of islands in the rivers and lakes, which influenced the backscattering coefficient of the water, so this part of water body is not extracted. In addition, due to the effect of noise and shadow of the mountain, some error may present in the extraction result (Figure 5).

The goal of object detection (or image classification) is usually a distinction between two classes (or object and background). Comparing the results of the automated extraction to reference data, an entity classified as an object as a True Positive (TP). A False Negative (FN) is an entity corresponding to an object in the reference that is classified as background, and a False Positive (FP) is an entity classified as an object that does not correspond to an object in the reference. A True Negative (TN) is an entity belonging to the background both in the classification and in the reference (Heipke C et al., 1997) (Zhan Q et al., 2005). The confusion matrix has a very simple structure. It consists of the number of entities assigned to these four classes (denoted by $\|\|$.$) .$ Two metrics for the quality of the results, the Completeness (Comp) and the Correctness (Corr), can be derived:

$$
\begin{aligned}
& \text { Comp }=\frac{\|T P\|}{\|T P\|+\|F N\|} \\
& \text { Corr }=\frac{\|T P\|}{\|T P\|+\|F P\|} \\
& \text { Quality }=\frac{\|T P\|}{\|T P\|+|| F P\|+|| F N\|}
\end{aligned}
$$

For validate the accuracy of the classification result, a shape files of water body was generated base on LANDSAT 8 data. This data was used to evaluate the quality of former classification results (Figure 6 and Table 1). True positive pixels are all the pixels in both datasets have the value of 1 . False Negative pixels are the pixels have value of 1 in knowledge-based digitizing dataset and value of 0 in classification results. False Positive pixels 
Vietnam Journal of Earth Sciences 37 (2015) 328-343

are the pixels have value of 0 in knowledge-based digitizing dataset and value of 1 in classification results. Table 1 shows pixel-based accuracy for the generated surface water bodies maps (with respect to the classified surface water bodies map that generated from Landsat 7 and Landsat 8 data).

Study site 1
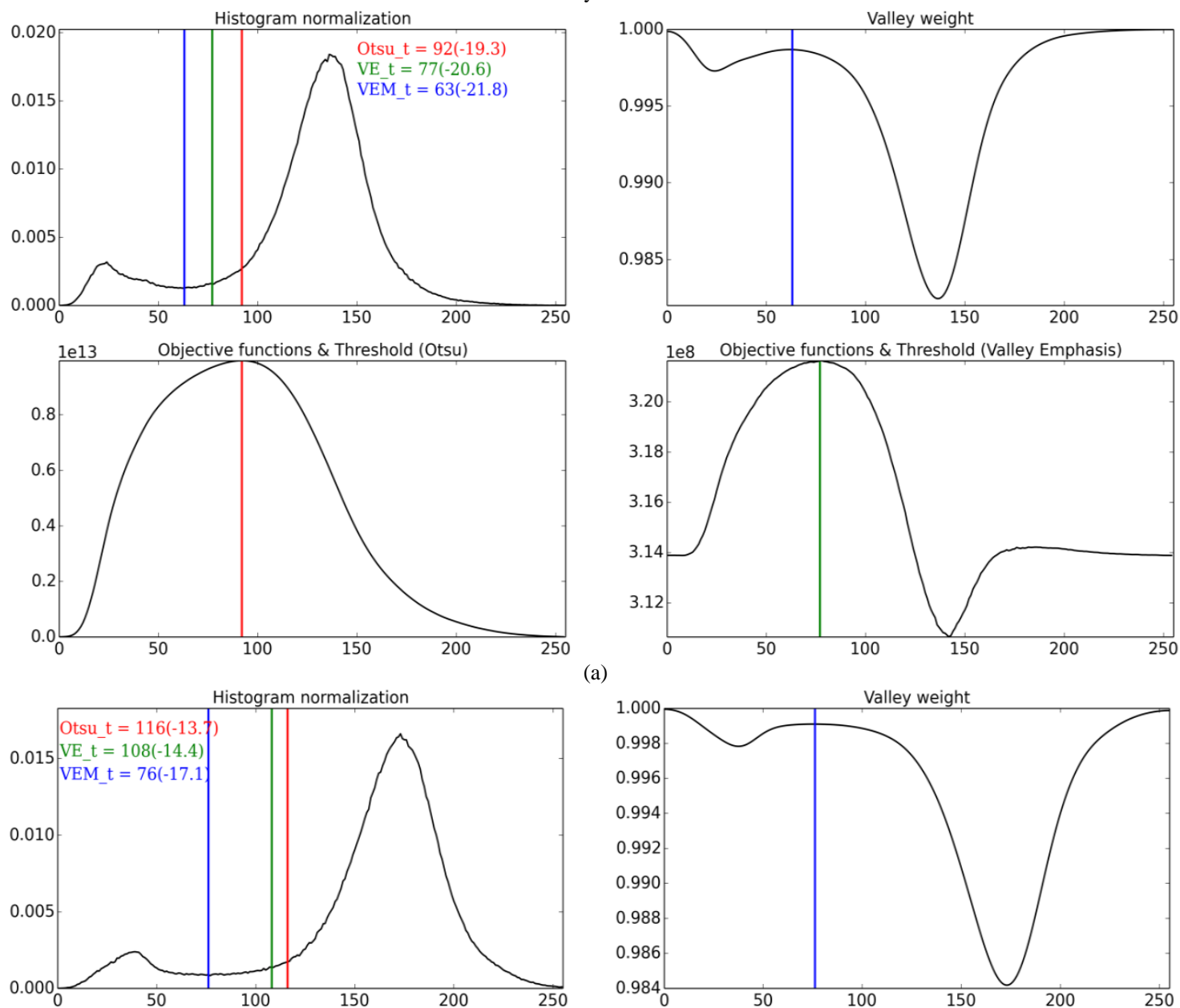

(a)
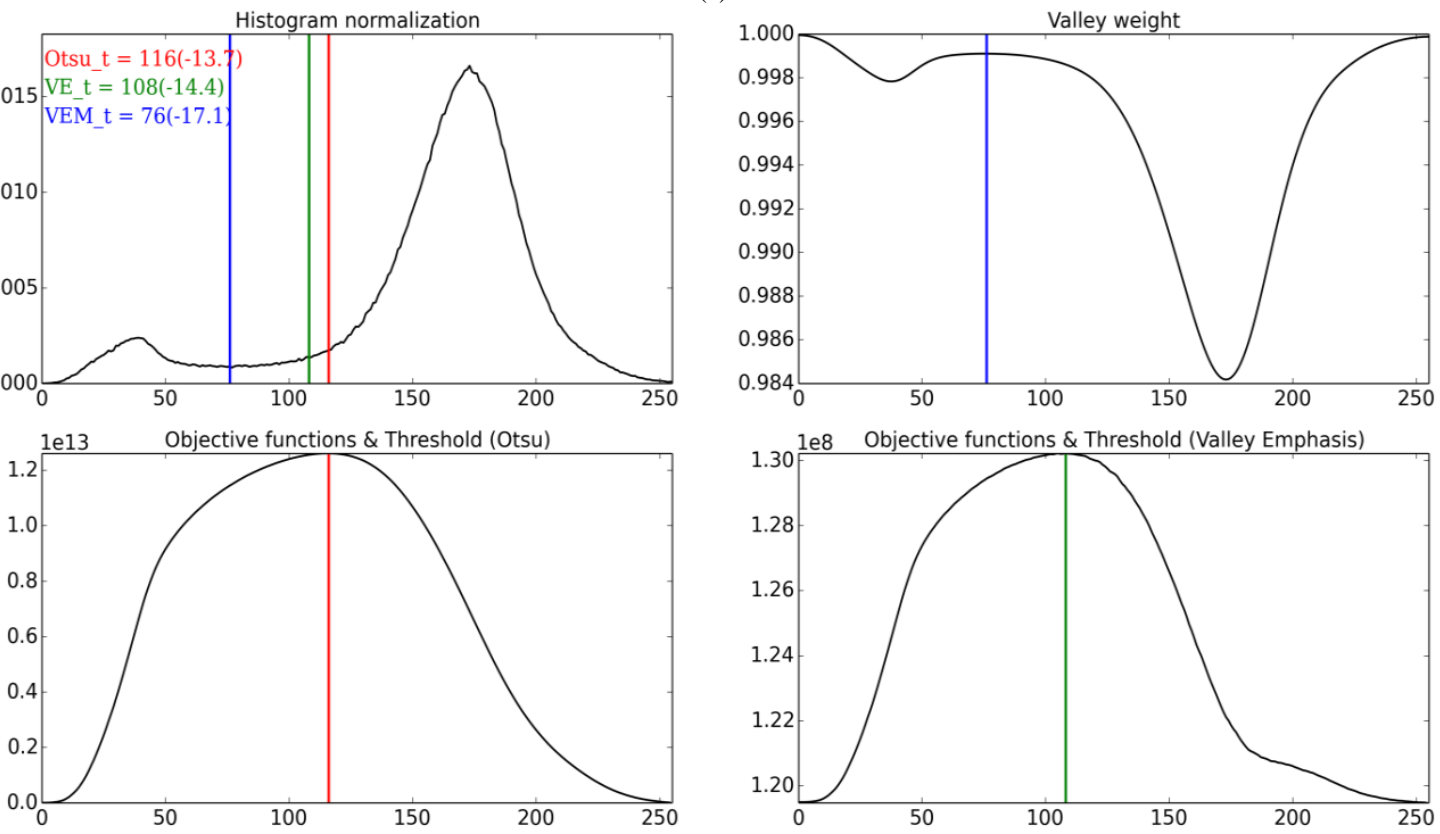

(b)

Fig. 4. Histogram, objective functions and threshold values from Otsu method, Valley- Emphasis method and a modified of ValleyEmphasis method 
N.B. Duy/Vietnam Journal of Earth Sciences 37 (2015)

Study site 2
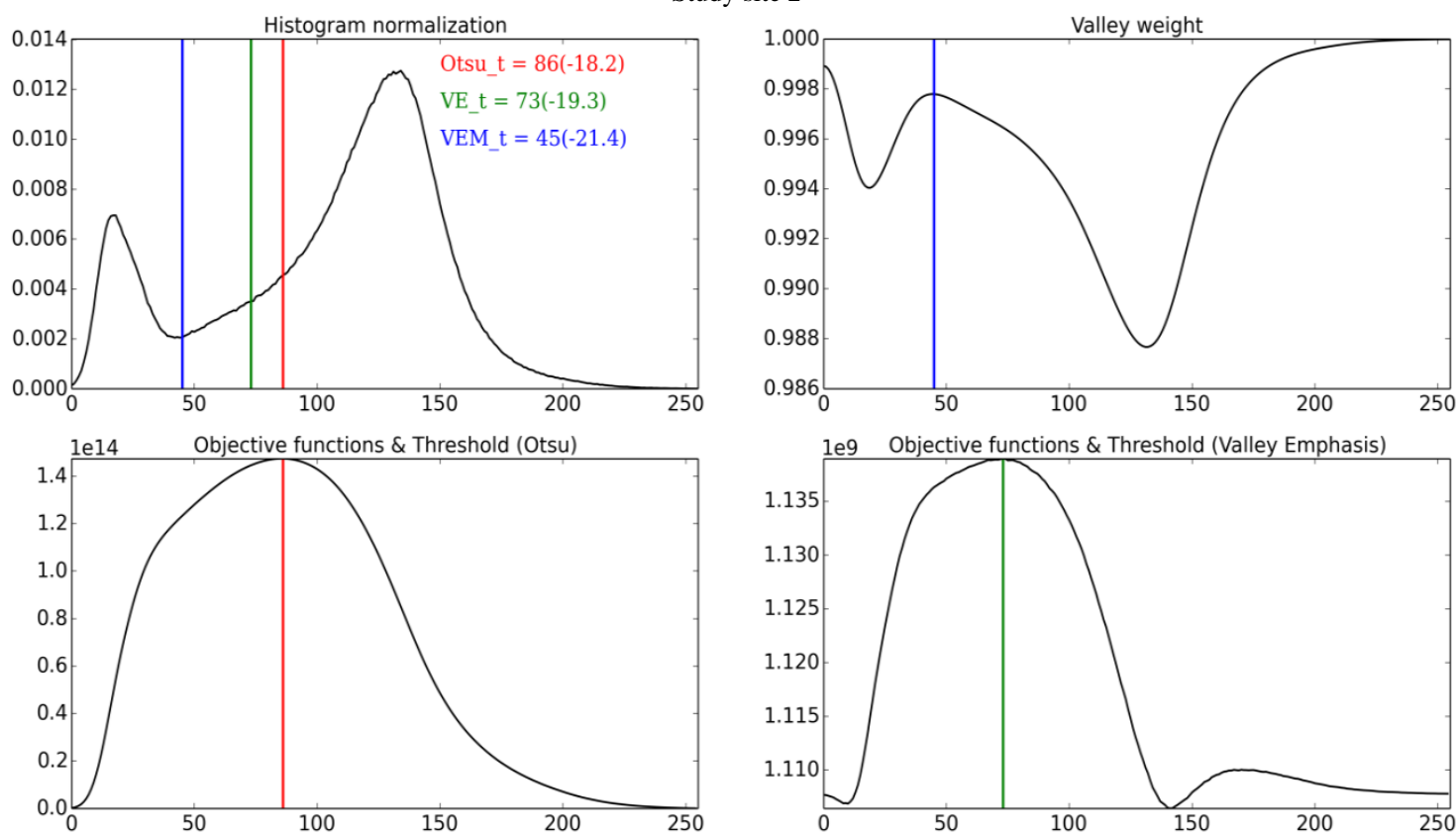

(c)
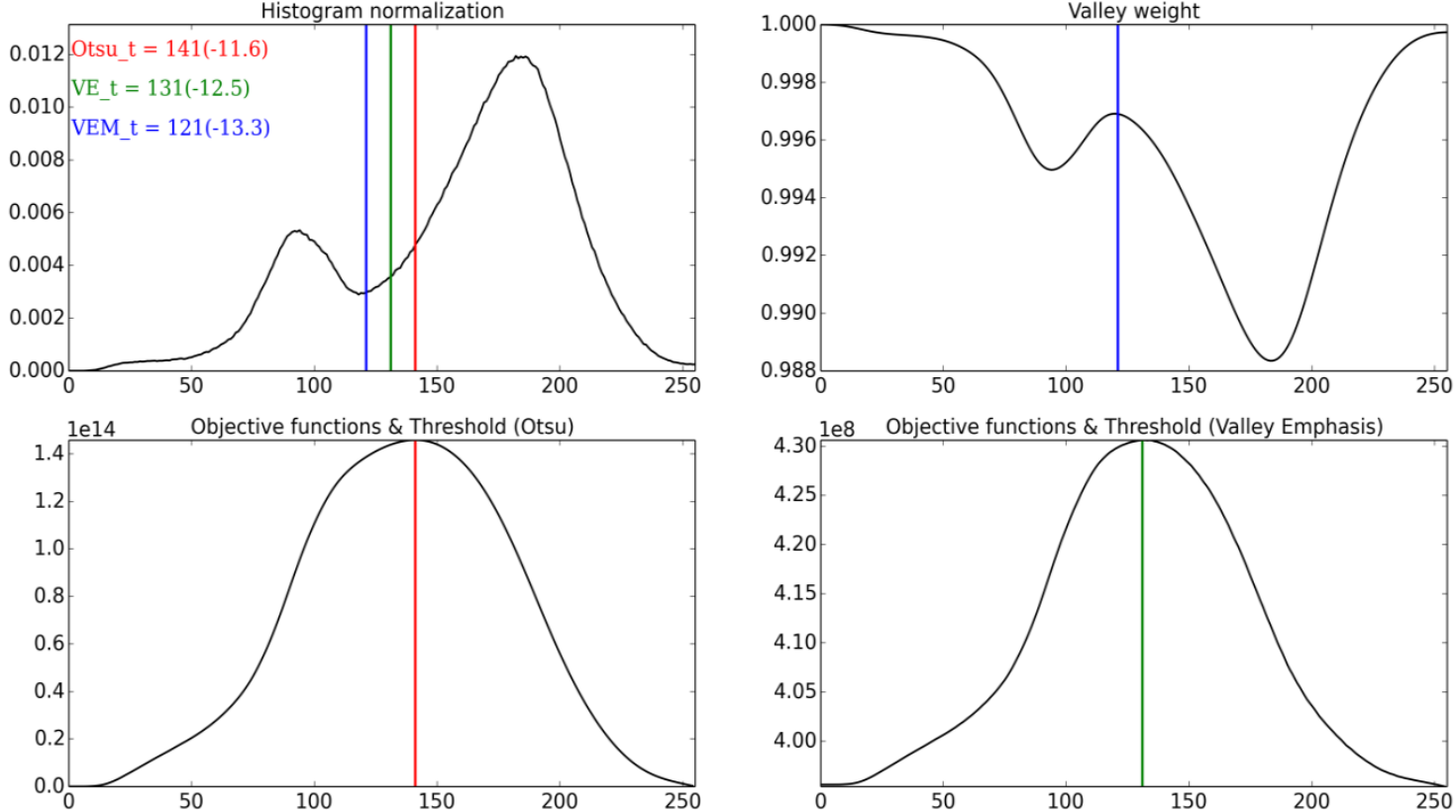

(d)

Fig. 4. $\rightarrow$ Continued page 338 
Vietnam Journal of Earth Sciences 37 (2015) 328-343

Study site 3
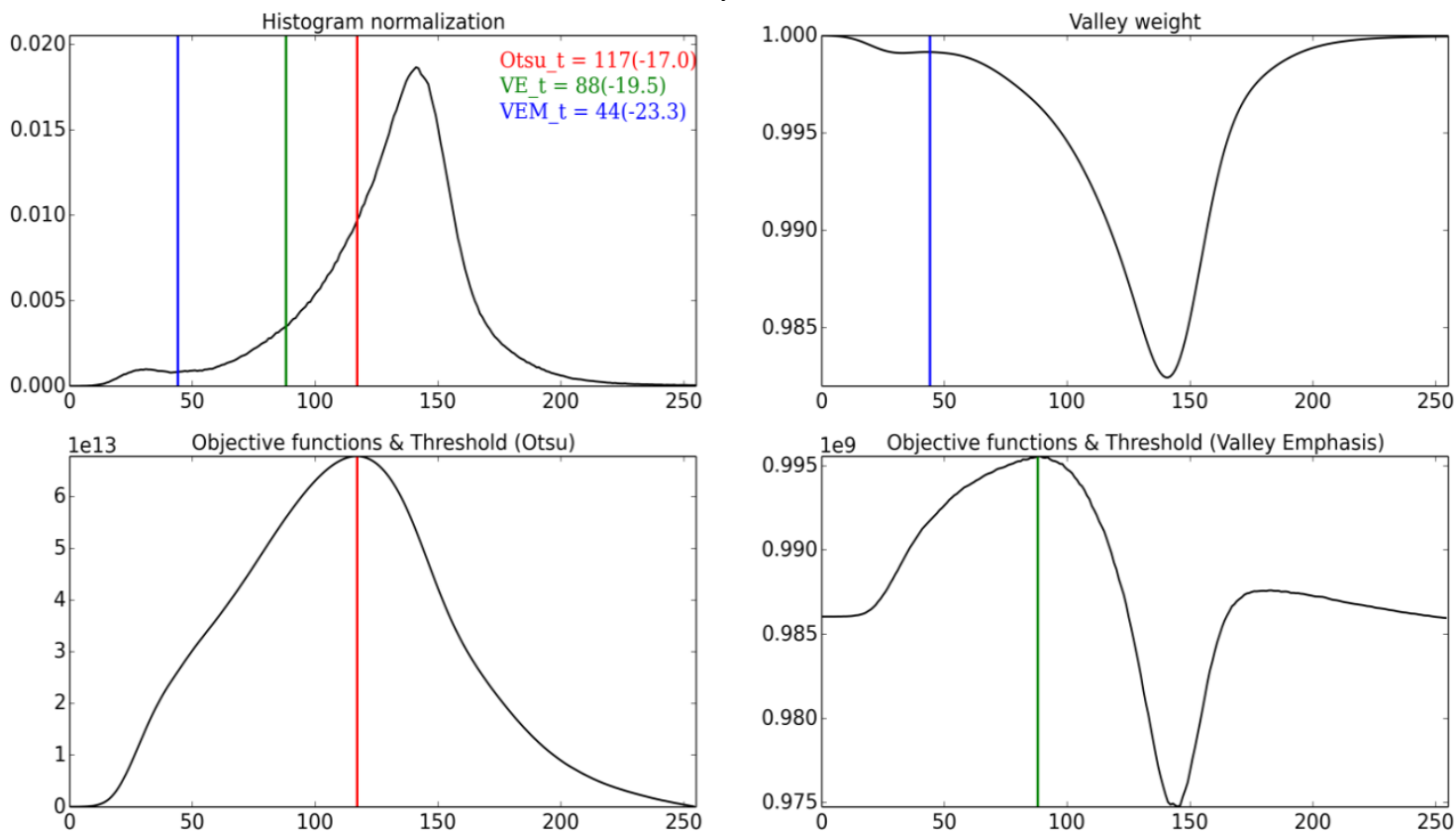

(e)
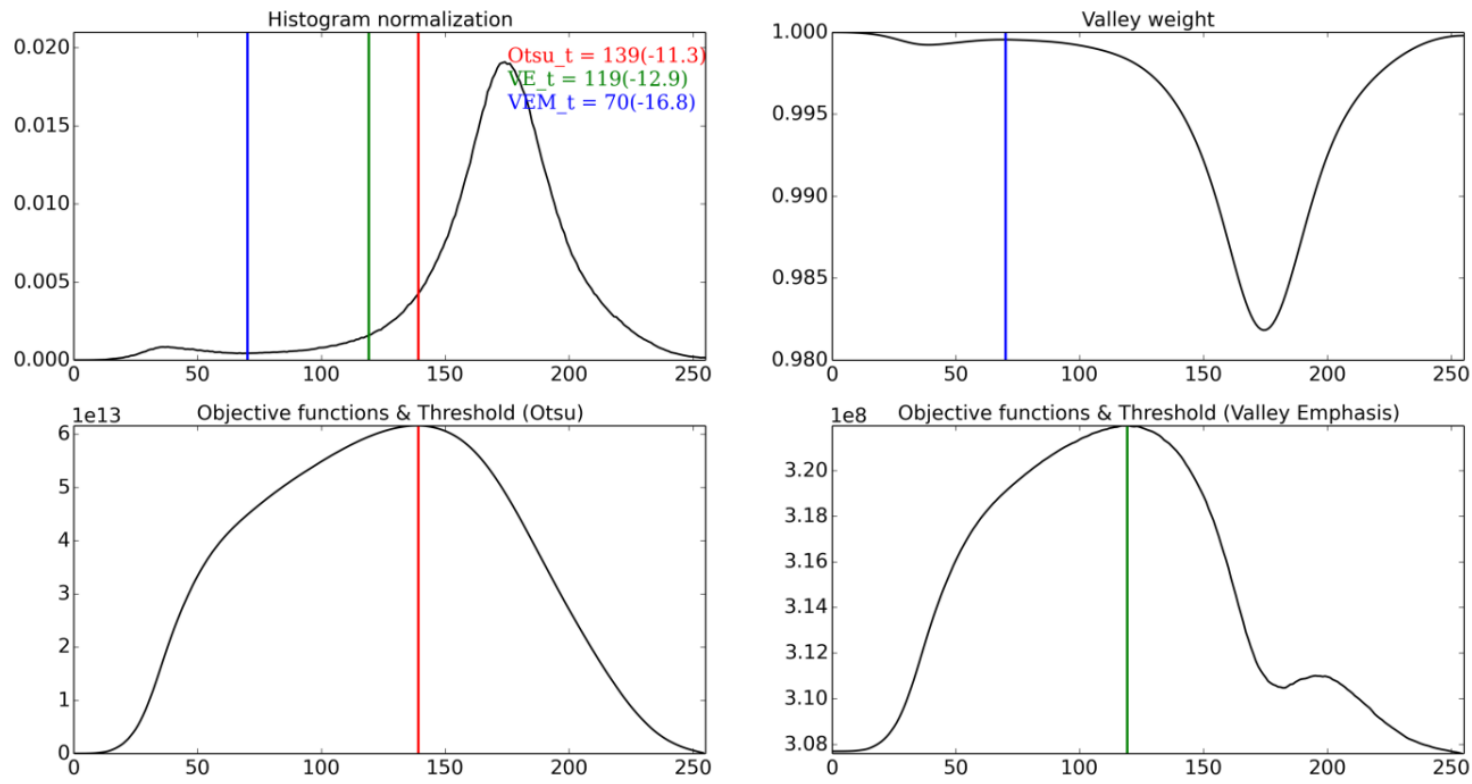

(f)

Fig. 4. 
N.B. Duy/Vietnam Journal of Earth Sciences 37 (2015)

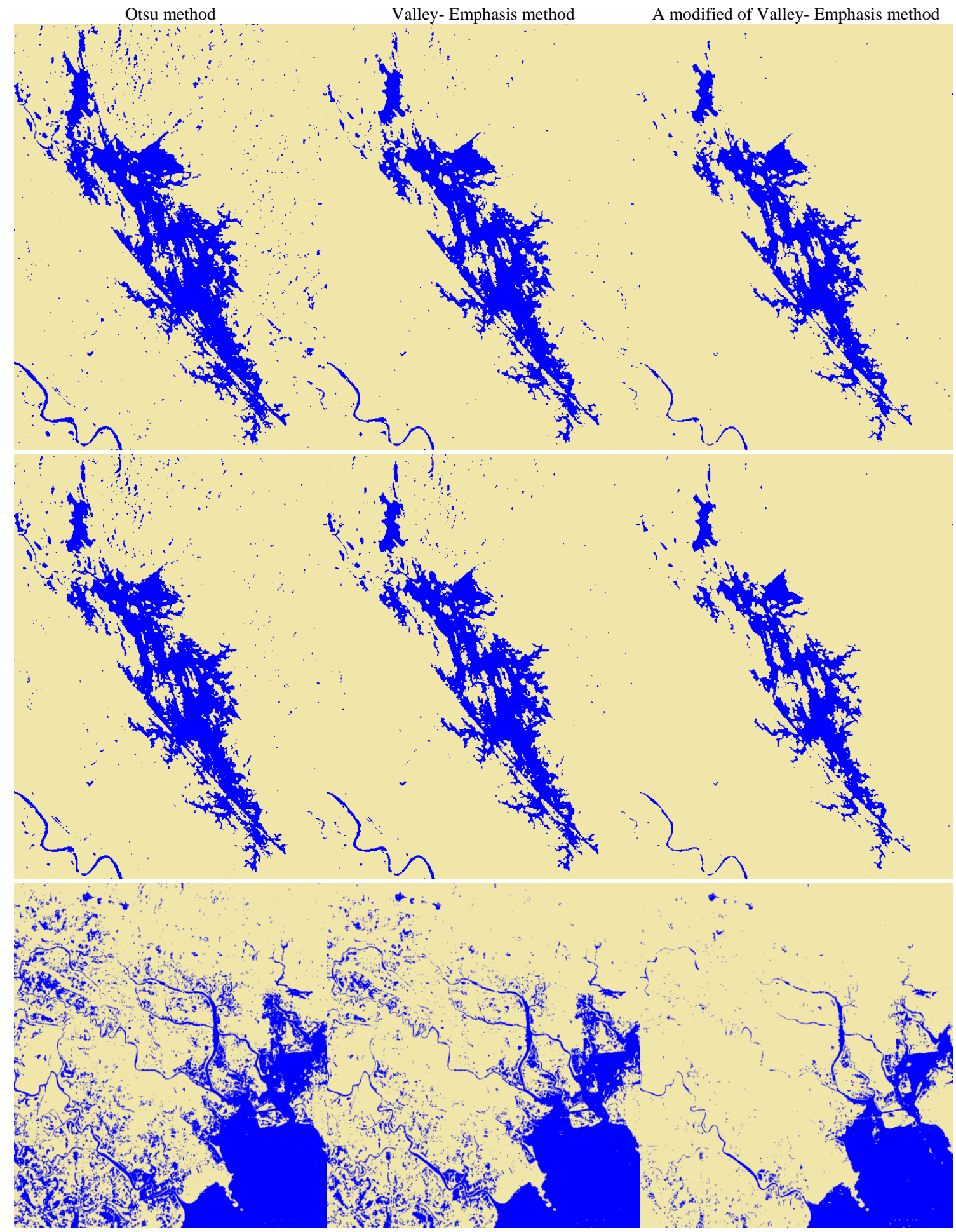

Fig. 5. Water body extraction (from VH polarization images) based on different threshold algorithms: Otsu method, ValleyEmphasis method and a modified of Valley- Emphasis method; $\rightarrow$ Continued page 340 
Vietnam Journal of Earth Sciences 37 (2015) 328-343
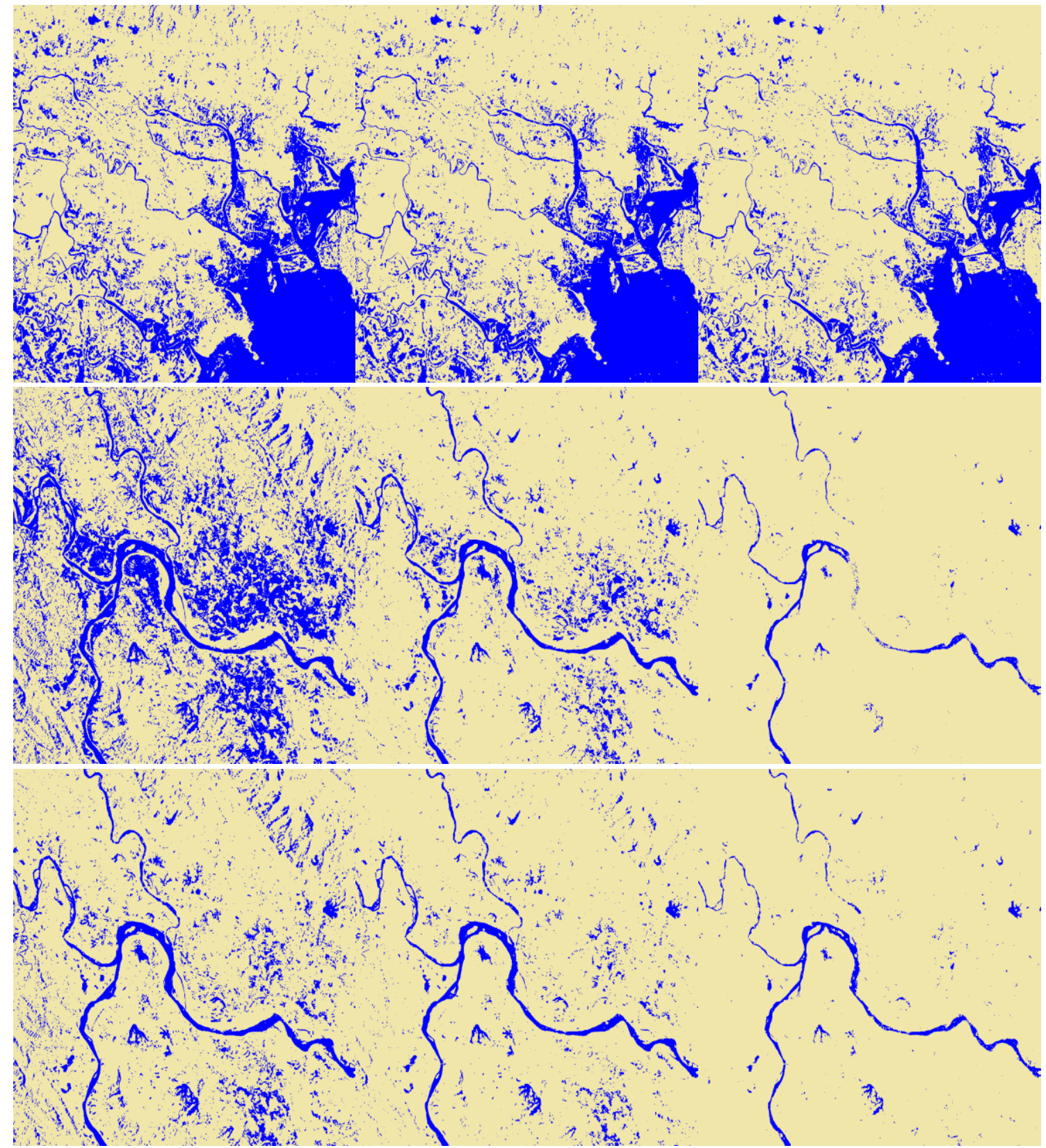

Fig. 5. 
N.B. Duy/Vietnam Journal of Earth Sciences 37 (2015)

Water body extraction from intersection of $\mathrm{VH}$ image and $\mathrm{VV}$ image
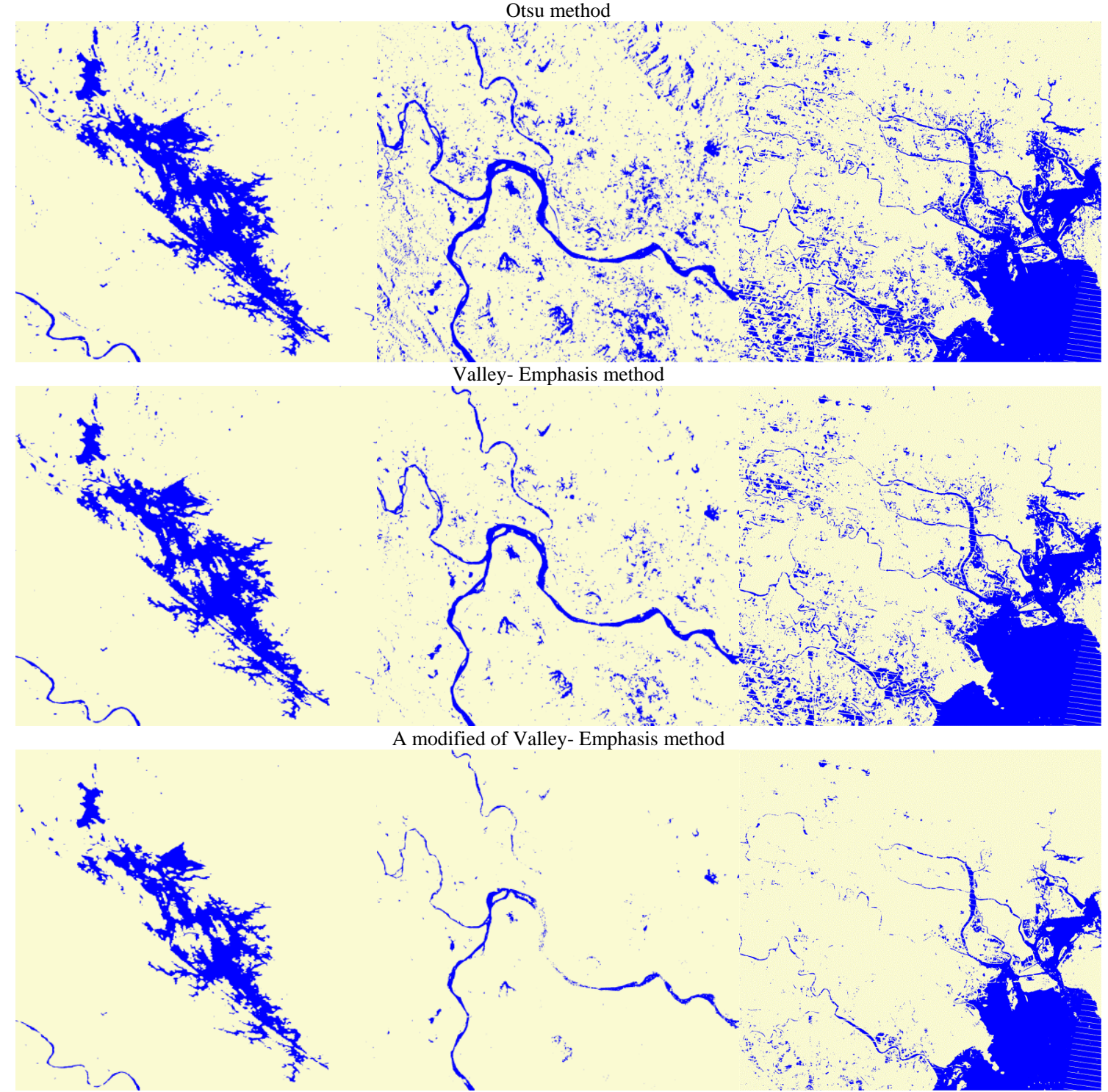

A modified of Valley- Emphasis method
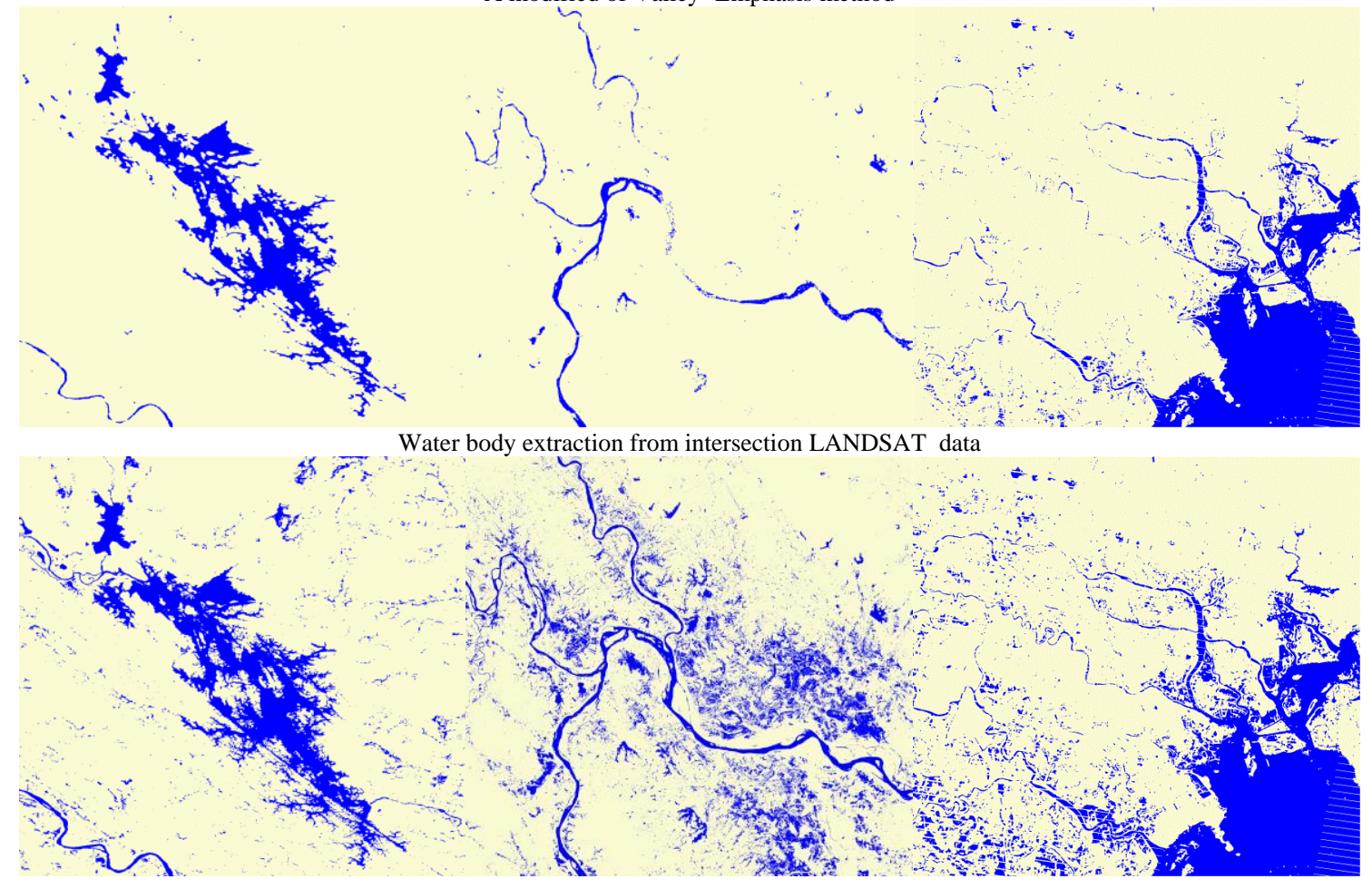

Fig. 6. Water body extraction (intersection of $\mathrm{VH}$ image and VV images) and Water body extraction from Landsat data 
Vietnam Journal of Earth Sciences 37 (2015) 328-343

Table 1. Accuracy assessment of the results

\begin{tabular}{|c|c|c|c|c|}
\hline$\overline{\text { Site }}$ & & Comp & Corr & Quality \\
\hline \multirow{3}{*}{1} & $\overline{\text { Otsu }}$ & $93.7 \%$ & $61.5 \%$ & $59.1 \%$ \\
\hline & Valley emphasis & & $68.3 \%$ & $54.1 \%$ \\
\hline & Valle & $81.8 \%$ & $80.6 \%$ & $68.3 \%$ \\
\hline \multirow{3}{*}{2} & Otsu & $66.5 \%$ & $45.7 \%$ & $1 \%$ \\
\hline & Valle & $53.0 \%$ & $71.2 \%$ & $43.7 \%$ \\
\hline & Valle & $24.5 \%$ & $92.1 \%$ & $24.0 \%$ \\
\hline \multirow{3}{*}{3} & Otsu & $95.2 \%$ & $85.7 \%$ & $85.5 \%$ \\
\hline & Valle & $98.8 \%$ & $90.7 \%$ & $89.7 \%$ \\
\hline & phasis 1 & $73.2 \%$ & $92.3 \%$ & $74.8 \%$ \\
\hline
\end{tabular}

\subsection{Discussion}

Research has demonstrated that Sentinel 1A SAR imagery can be used to accurately extract surface water bodies with high accuracy. Reference data investigation of the study area shows that there is a lot of vegetation growing on the water surface (Study site number 2, Figure 6). In addition, many dams, fences and other artificial construction objects were located in the shrimp ponds (Study site number 1 and study number 3, Figure 6). A variety of features distributing in water bodies make it difficult to interpret. This brought about the difficulty of classification. Unlike surface water bodies, the brightness values of vegetation on the water on SAR imagery were much higher than those of water. So they can be easily divided into non-water bodies by mistake. The radar is sensitive to the open water bodies surface. By using Sentinel 1A SAR images, surface water bodies can be extracted effectively, even many small water bodies such as small ponds.

As can be seen in the Table 1, the valleyemphasis method outperformed the other methods for all three study sites. This is a fast and more accurate way for extracting water bodies. The low classification accuracy in the study site number 2 for all the methods due to data acquisition time are different (02 July, 2015 for Sentinel 1A SAR data and 01 July, 2015 for Landsat 7 data). The open water areas and small water bodies can be extracted using Sentinel 1A SAR images more effectively.

The survey found that many emergent plants growing in ponds as well as along rivers and in the agricultural fields. They are easy to be confused with the flooded-vegetation areas with great backscattering values due to their double-bounce scattering from smoothed surface areas (water) mixed with vegetation areas. Meanwhile, the study also found that Landsat images are not sensitive to the small water bodies and they can be easily classified by mistake as those types of surrounding features. As a result, many of the small water bodies are omitted when using the Landsat images for the extraction of water information.

\section{Conclusions}

From this study, the ability of the image thresholding methods to improve the extraction of surface water body was tested based on dual polarization Sentinel 1A SAR imagery over three study areas in the central and eastern of Northern Vietnam. The optimum threshold values for the delineation of water bodies using different thresholding algorithms is derived for $\mathrm{VV}$ and $\mathrm{VH}$ polarizations. In $\mathrm{VH}$ polarization, the threshold varies are very well located in the valley of the histogram for all the preformed algorithms. $\mathrm{VH}$ polarization have better potential to differentiate water bodies. However, combining the water bodies layers derived from $\mathrm{VV}$ and $\mathrm{VH}$ polarizations the water body areas match closely to the classification results of optical data.

Compared with the visible and multi-spectral remote sensing data, Sentinel-1A SAR has broader application prospects in the flood disaster assessment due to its all-time and all- weather data acquisition capability, and not affected by rainy and cloudy factor. Otsu method, the valleyemphasis method and a modified valley-emphasis method were applied for surface water body extraction from Sentinel-1A SAR images captured three study sites in NE Vietnam. The Valley Emphasis method outperformed the optimal thresholding method for most SAR backscattering images in all study areas. Further applications are required to assess the robustness of the method on imagery from other SAR sensors and other areas of the world.

The acquired results proven to be highly worthy. The employed method is simple and easy to implement. It improves the automatic identification level of water body identification to a large extent.

However, there are several difficulties associated with surface water body extraction from Sentinel-1A SAR satellite imagery using image 
N.B. Duy/Vietnam Journal of Earth Sciences 37 (2015)

thresholding methods, including: (1) inefficient identification of mixed water pixels; (2) confusion of water bodies with background noise; and (3) variations in the optimal water extraction threshold that depend on the characteristics of an individual location. In addition, pixels in the shadow of the mountain also has low scattering coefficient, therefore, the results of water body extraction often include the shadow of the mountain.

\section{Acknowledgments}

The authors are highly grateful to the European Space Agency (ESA) for support, encouragement and data access for this study.

\section{References}

Otsu, N., 1979: A threshold selection method from gray-level histograms, IEEE Trans Sys, Man Cyber, 9 (1), pp. 62-66.

Hui-Fuang, Ng., 2006: Automatic thresholding for defect detection," Pattern Recognition Letters, vol. 27, pp. 1644-1649.

Lun, F. Bo, L., 2012: A modified valley-emphasis method for automatic thresholding, Pattern Recognition Letters,
Volume 33, Issue 6, pp. 703-708.

Small, D., 2011: Flattening gamma: radiometric terrain correction for SAR imagery, IEEE Transactions on Geoscience and Remote Sensing 49, pp. 3081-3093.

Gonzalez, C. Woods, R., 2002: Digital Image Processing, Prentice Hall.

Ye, Z., Mohamadian, H., Ye, Y., 2008: Grey Level Image Processing Using Contrast Enhancement and Watershed Segmentation with Quantitative Evaluation. International Workshop on Content-Based Multimedia Indexing, pp. $470-475$.

Zhang, J., Zheng, J., Cai, J., 2010: A Diffusion Approach To Seeded Image Segmentation, IEEE Conference On Computer Vision And Pattern Recognition (CVPR), San Francisco, USA, Jun. 13-18, pp. 2125- 2132.

Heipke, C., Mayer, H., Wiedemann, C., Sensing, R., Jamet O., 1997: Evaluation of Automatic Road Extraction. Photogramm. Remote Sens, pp. 47-56.

Zhan, Q., Molenaar, M., Tempfli, K., Shi, W., 2005: Quality assessment for geo-spatial objects derived from remotely sensed data. Int. J. Remote Sens, 26-14, pp. 2953-2974. 